Ann. Biol. anim. Bioch. Biophys., I969, 9 (3), 435-440.

\title{
UN NOUVEAU DISPOSITIF DE PERFUSION POUR L'ETUDE DE L'ABSORPTION INTESTINALE IN VITRO
}

\author{
R. ROZEN \\ avec la collaboration technique de W. LorSEL \\ Station de Recherches sur les Aliments de l'Homme, \\ Centre de Recherches agronomiques de Dijon \\ Institut national de la Recherche agronomique
}

\section{SOMMAIRE}

Un nouveau dispositif permettant la perfusion in vitro d'anses intestinales isolées est décrit. La circulation du liquide dans la lumière intestinale se fait en circuit ouvert ; la circulation du liquide du côté de la séreuse se fait en circuit fermé. Le mouvement des solutions est assuré par une pompe péristaltique à débit constant.

\section{INTRODUCTION}

L'étude des modalités de l'absorption intestinale a fait de rapides progrès depuis la démonstration par FISHER et PARSONS (I949), qu'il est possible de maintenir en activité un intestin isolé. Ces auteurs ont décrit le premier appareil utilisant une circulation de solution saline dans une anse intestinale de Rat non retournée, et 1'ont utilisé pour étudier l'absorption des sucres et des acides aminés. Cet appareil comprend deux réservoirs de Ioo $\mathrm{ml}$ contenant des solutions " muqueuse " et " séreuse ". La circulation des solutions est assurée par des bulles de gaz (oxygène, carbogène ou azote selon les expériences) qui poussent les liquides (gas-lift) et une pression hydrostatique de $35 \mathrm{~cm}$ d'eau. Il en résulte une circulation rapide de 35 à $45 \mathrm{ml} / \mathrm{mn}$, non régulière et difficilement contrôlable. Les deux circuits " muqueux " et "séreux" sont des circuits fermés. LEE ( 1963 ), dans une étude sur le rôle des vaisseaux lymphatiques mésentériques dans l'absorption intestinale de l'eau, utilise le même dispositif légèrement modifié : la circulation de la solution " muqueuse » est assurée par une pompe ; la solution " séreuse " ne circule pas.

WISEMAN (r953) a décrit un autre appareil de perfusion intestinale pour anses 
isolées, dans lequel 3 segments intestinaux mis en parallèle sont perfusés ensemble. Le principe de fonctionnement est cependant identique à celui de l'appareil de FISHER et PARSONS. Diverses modifications au dispositif de WISEMAN ont été apportées par différents auteurs, pour des usages particuliers. Ainsi, SMYTH et WHALER (I953) améliorent l'oxygénation du liquide interne et ajoutent un collecteur de $\mathrm{CO}_{2}$ pour étudier la décarboxylation des acides aminés. SMYTH et TAYLOR (I957) suppriment la solution saline du côté séreux afin d'étudier le transport actif de l'eau : le liquide transporté est collecté dans un tube adapté à l'appareil de SMYTH et WHALER.

DARLINGTON et QUASTEL (I953) ont proposé un appareil de perfusion plus simple que les précédents, avec lequel ils ont montré que le système respiratoire de la muqueuse intervenait dans le processus d'absorption du glucose. RIKLIS et QUASTEL, (I958) avec le même appareil ont étudié le rôle du potassium dans l'absorption du glucose. Tous ces appareils mettent en jeu de grandes quantités de liquides, environ roo $\mathrm{ml}$ du côté muqueux et autant du côté séreux.

CSAKY et THALE (I960) ont mis au point une technique simple d'étude du transport actif du glucose par 1'intestin isolé de Crapaud, technique dans laquelle les volumes des compartiments muqueux et séreux sont très réduits : $14 \mathrm{ml}$ de solution du côté de la muqueuse et Io $\mathrm{ml}$ du côté de la séreuse. Un même souci de réduction des volumes liquidiens mis en jeu s'observe dans l'appareil de SAHAGIaN et coll. (I967).

Tous les dispositifs cités diffèrent les uns des autres par des détails, mais non dans leurs principes:

I. La circulation des solutions salines et assurée par le "gas-lift." La vitesse de circulation est extrêmement rapide (de 35 à $45 \mathrm{ml} / \mathrm{mn}$ ), non régulière et difficilement contrôlable.

2. La circulation de la solution muqueuse (et de la solution séreuse quand elle existe) se fait en circuit fermé, c'est-à-dire que le même liquide vient au contact de l'intestin plusieurs fois ; la concentration des produits étudiés varie donc à tout instant.

3. Sauf dans le dispositif de Csaky et ThaLE, le segment intestinal utilisé est en position verticale. Les anses ont de $\mathrm{I} 8$ à $20 \mathrm{~cm}$ de long ; la pression varie donc du haut en bas de l'anse de façon importante. Or, SMYTH et TAYLOR (r957) ont montré que l'accroissement de la pression hydrostatique provoquait des variations dans l'absorption de l'eau.

Pour ces diverses raisons, nous avons mis at point un nouvel appareil en tenant compte des réalisations déjà existantes.

Les principes qui nous ont guidé sont les suivants :

- les circulations des solutions " muqueuse " et "séreuse " sont indépendantes l'une de l'autre et contrôlées en permanence ;

- le circuit “ muqueux » est un circuit ouvert, une pompe apportant à l'intérieur de l'anse une solution de concentration définie et constante ; le perfusat est recueilli à la sortie de l'anse de façon continue. Le circuit "séreux » est un circuit fermé, ce qui assure la concentration des corps absorbés ;

- 1a vitesse d'évacuation est très lente du côté " muqueux " et au contraire rapide du côté " séreux ", afin d'assurer une bonne homogénéisation de la solution " séreuse";

- l'anse intestinale utilisée est en position horizontale. 


\section{DESCRIPTION DU DISPOSITIF}

La figure i donne un schéma de l'appareil.

Un tube de verre $\mathrm{A}$ de $20 \mathrm{~cm}$ de long et $\mathrm{I} \mathrm{cm}$ de diamètre, ouvert aux 2 extrémités, est manchonné; dans le manchon circule très rapidement de l'eau à $37^{\circ}$. Ce tube est percé de 3 orifices :

- l'un central $(\mathrm{O})$ de $2 \mathrm{~cm}$ de diamètre,

- deux latéraux ( $\mathrm{E}$ et $\left.\mathrm{E}^{\prime}\right)$ de $0,3 \mathrm{~cm}$ de diamètre.

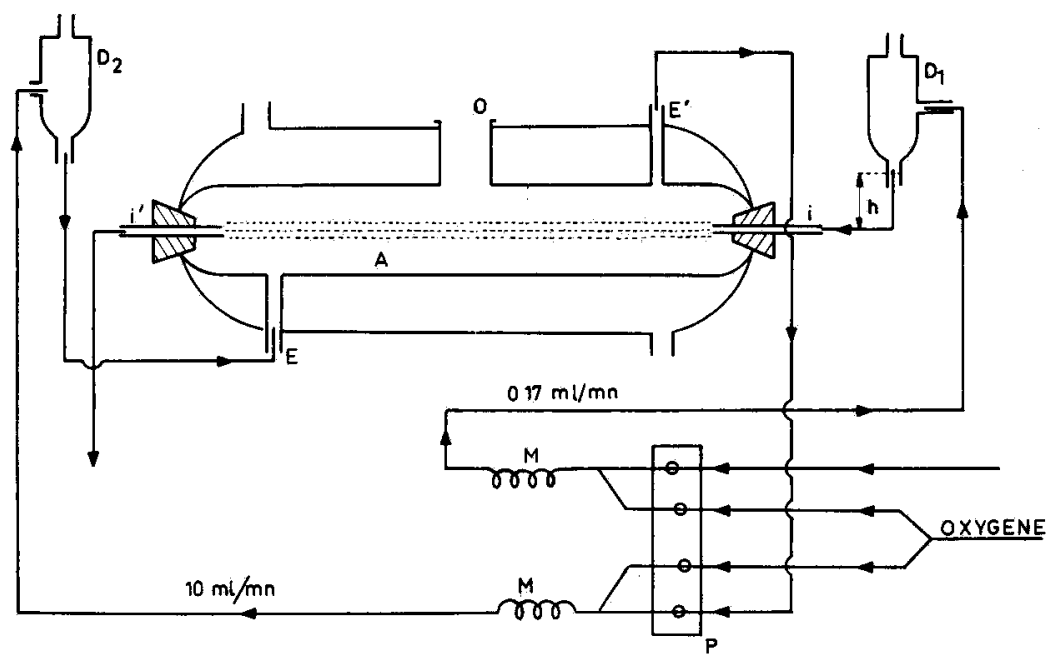

FIG. I.

Un segment d'intestin grêle de rat de $20 \mathrm{~cm}$ de long environ, non retourné, canulé aux deux extrémités ( $\mathrm{I}$ et $\mathrm{I}^{\prime}$ ) est placé dans le tube $\mathrm{A}$ et fixé par 2 bouchons.

Avec des tubes en tygon calibrés (tubes "Technicon ") on forme deux circuits indépendants:

- un circuit externe (pour la solution " séreuse »), fermé :

$$
\mathrm{E} \rightarrow \mathrm{E}_{2}^{\prime} \rightarrow \mathrm{P} \rightarrow \mathrm{D}_{2} \text { de } \text { ro } \mathrm{ml} / \mathrm{mn}
$$

- un circuit interne (pour la solution " muqueuse ") ouvert :

$$
\mathrm{P} \rightarrow \mathrm{D}_{1} \rightarrow \mathrm{I} \rightarrow \mathrm{I}^{\prime} \text { de } 0, \mathrm{I} 7 \mathrm{ml} / \mathrm{mn} \text { (soit Io } \mathrm{ml} / \mathrm{H} \text { ) }
$$

$20 \mathrm{ml}$ de solution " séreuse " sont introduits rapidement en $\mathrm{O}$. Une pompe péristaltique $\mathrm{P}$ à débit constant (modèle Technicon) établit la circulation des fluides.

La gazéification des solutions " séreuse " et " muqueuse " est assurée par la pompe $\mathrm{P}$ et des mélangeurs en verre $M$ (modèle Technicon). Ces solutions sont " débullées " en $D_{1}$ et $D_{2}$ juste avant leur entrée dans le tube $A$. Les « débulleurs « $\mathrm{D}_{1}$ et $\mathrm{D}_{2}$ servent également au contrôle des pressions hydrostatiques à l'intérieur et à l'extérieur de l'anse intestinale $(\mathrm{h})$.

La prise des échantillons à analyser se fait par la canule de sortie de l'anse intestinale I' en ce qui concerne le liquide " muqueux ", par l'orifice O en ce qui concerne le liquide " séreux ». Pour modifier le moins possible le liquide séreux qui circule en circuit fermé, la prise d'essai est de $0,2 \mathrm{ml}$ toutes les Io minutes.

\section{ESSAIS PRÉLIMINAIRES}

En vue d'étudier ultérieurement les modalités d'absorption du glucose par l'intestin grêle, nous avons fait quelques expériences portant sur la vitesse de disparition du glucose dans la lumière intestinale. 


\section{A. - Technique opératoire}

Un rat de $250 \mathrm{~g}$ environ, à jeûn depuis $24 \mathrm{~h}$, est décapité. Un segment de jéjunum de $20 \mathrm{~cm}$ de long environ est prélevé selon la technique classique (DarLINGToN et QUASTEL, I953), il est rapidement nettoyé avec une solution de Krebs-Ringer bicarbonatée et non glucosée. Les canules sont fixées aux deux extrémités de l'anse, le tout est placé à l'intérieur du tube, et rattaché au circuit de perfusion. Il faut prendre soin de respecter le sens physiologique d'écoulement des liquides dans l'intestin, afin d'éviter tout anti-péristaltisme. Entre la mort de l'animal et 1'introduction du segment intestinal dans 1'appareil, il s'écoule 5 à 7 minutes au maximum.

Dès que l'intestin est en place, la solution en contact avec la séreuse est introduite par 1'orifice central du tube. Il s'agit de $20 \mathrm{ml}$ de solution de Krebs-Ringer, bicarbonatée, dans laquelle on ajoute du glucose de façon à obtenir une concentration de 5 millimôles par litre. Dans le cas présent, la même solution est perfusée du côté muqueux de la paroi intestinale.

La gazéification des solutions est assurée par du carbogène $\left(5 \mathrm{p}\right.$. Ioo $\mathrm{CO}_{2}, 95$ p. $\mathrm{I0O} \mathrm{O}_{2}$.)

La pompe est mise en marche ; on attend 5 minutes pour l'équilibration de tout le système, les premières gouttes de liquide "muqueux " ayant traversé l'intestin sont rejetées, et le temps "zéro " de l'expérience commence. Nous notons toujours le temps écoulé entre la mort de 1'animal et ce temps "zéro ". S'il dépasse I5 à I9 mn, nous n'effectuons pas d'expérience.

\section{B. - Application d̀ l'étude de l'absorption du glucose}

Une vitesse de circulation de Io $\mathrm{ml} / \mathrm{h}$ du liquide " muqueux " a été choisie après quelques expériences préalables qui nous ont montré que l'absorption d'eau par l'intestin est relativement indépendante de la vitesse de perfusion lorsque celle-ci varie de Io à $40 \mathrm{ml} / \mathrm{h}$. En dessous de Io $\mathrm{m} / \mathrm{h}$ l'absorption d'eau diminue. L'augmentation de la vitesse de perfusion provoque la desquamation de cellules intestinales et du mucus abondant dans le perfusat recueilli.

La circulation du liquide "séreux" est toujours de $600 \mathrm{ml} / \mathrm{h}$.

En plaçant des deux côtés de la paroi intestinale une solution identique de Krebs-Ringer bicarbonaté, contenant du glucose en concentrations croissantes, nous avons étudié la disparition du glucose de la lumière intestinale.

Le dosage du glucose est effectué par la technique enzymatique à la glucose oxydase (1) modifiée pour stabiliser la coloration (GUIDotTr, Corombo et FoA (I96I).

Le calcul de la quantité disparue se fait de la manière suivante :

$\mathrm{A}=$ quantité de glucose $(\mathrm{en} \mu \mathrm{M})$ offerte en $5 \mathrm{mn}$ (concentration $\times$ débit).

$\mathrm{B}=$ quantité du glucose (en $\mu \mathrm{M})$ retrouvée dans le liquide muqueux pendant le même temps (concentration $\times$ quantité de liquide recueilli à la sortie de l'anse).

Glucose disparu du liquide muqueux en $5 \mathrm{mn}: \mathrm{A}-\mathrm{B}$.

Les mesures sont faites toutes les 5 minutes pendant 40 minutes. Les résultats sont représentés dans la figure 2 .

(1) Réactif des Laboratoires Biotrol, Paris. 
On voit que, si à basse concentration $(5 \mu \mathrm{M} / \mathrm{ml})$ la vitesse d'absorption est constante dans le temps, aux concentrations moyennes (jusqu'à $30 \mu \mathrm{M} / \mathrm{ml}$ ) cette vitesse d'absorption tend à diminuer avec le temps. Dans l'hypothèse d'un transport teur de glucose de nature enzymatique (CRANE, I960) ces courbes s'expliquent par une saturation progressive du transporteur.

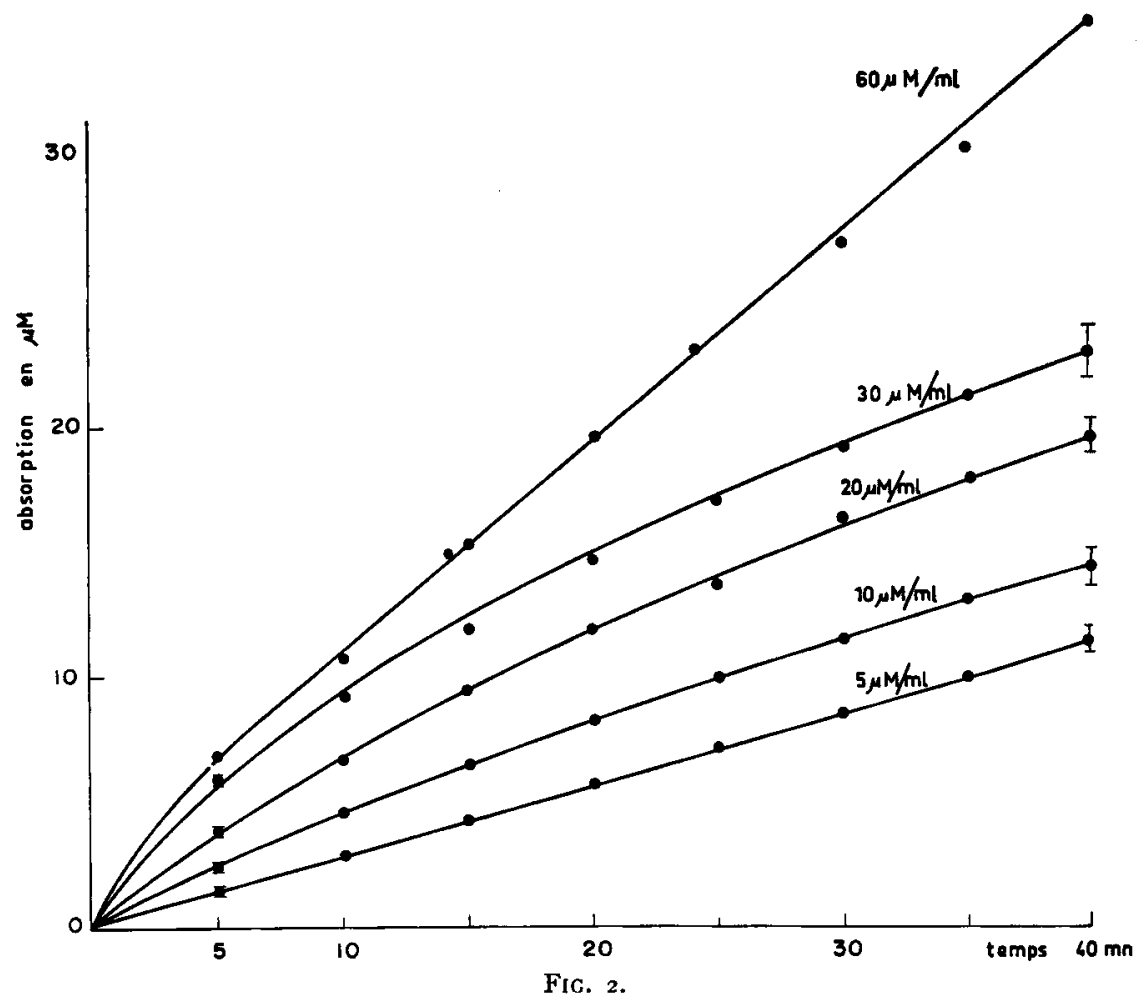

Aux très fortes concentrations $(60 \mu \mathrm{M} / \mathrm{ml})$ un phénomène complexe semble intervenir dans le transport du glucose. CATIER (I965) faisant ingérer des solutions de glucose à des chiens porteurs de fistules intestinales, observe également qu'aux fortes concentrations (supérieures à $45 \mu \mathrm{M} / 1$ ) l'absorption ne suit plus la loi de MichaelisMenten. L'absorption passive, dépendant uniquement de la concentration intraluminale du glucose, semble primer sur l'absorption active dépendant du transporteur. Ceci peut s'interpréter soit comme un débordement des processus de régulation de l'absorption du glucose, soit comme la conséquence de modifications morphologiques (gonflement et élargissement des pores et de 1a membrane) qui permettraient un accroissement de la diffusion (CATIER, I965).

Nous dirons, pour conclure, qu'il est nécessaire de choisir soigneusement la concentration du glucose, et probablement de tous les corps absorbés activement, selon le type de recherche que l'on se propose d'effectuer. 


\section{SUMMARY}

A NEW APPARATUS FOR THE " IN VITRO " STUDY OF INTESTINAL ABSORPTION

A new apparatus for the in vitro perfusion of isolated intestinal loops is described. The fluid circulating in the intestinal lumen is in an open circuit ; the fluid circulating on the serosal surface is in a closed circuit. The fluids are kept circulating using a peristaltic pump.

\section{RÉFÉRENCES BIBIIOGRAPHIQUES}

Catier J., I965. Absorption intestinale du glucose. These de doctorat en Médecine (Marseille).

Crane R. K., 1960. Intestinal absorption of sugars. Physiol. Rev., 40, 789-825.

Craky T. Z., Thale M., 1960. Effect of ionic environment on intestinal sugar transport. J. Physiol., 151, 59-65.

DaRlington W. A., Quastel. J. H., I953. Absorption of sugars from isolated surviving intestine. Arch. Biochem., 43, 194-207.

Fisher R. B., Parsons D. S., 1949. Glucose absorption from surviving rat small intestine. J. Physiol., $110,281-293$.

Guidotti G., Colombo J. P., Foa B. P., 1961. Enzymatic determination of glucose. Stabilization of color developped by oxydation of $o$-dianisidine. Anal. Chem., 33, I5I-I 53.

LEE J. S., 1963. Role of mesenteric lymphatic system in water absorption from rat intestine in vitro. Am. J. Physiol., 204, 92-96.

RikLis E., QuASTEL J. H., 1958. Effects of cations on sugar absorption by isolated surviving guinea pig intestine. Canad. J. Biochem. Physiol., 36, 347-362.

Sahagian B. M., Harding-Barlow I., PerRy H. M., 1967. Transmural movements of zinc, manganese, cadmium and mercury by rat small intestine. J. Nutr., 98, $291-299$.

SMYTH D. H., TAYLOR C. B., 1957. Transfer of water and solutes by an in vilro intestinal preparation. J. Physiol., 136, 632-648.

SMYTh D. H., Whaler B. C., 1953. Apparatus for the in vitro study of intestinal absorption. J. Physiol. 121, 2 p.

Wiseman G., 1953. Absorption of amino acids using an in vitro technique. $J$. Physiol., 120, 63-72. 